\section{Evaluation of Drug Candidates for Preclinical Development: Pharmacokinetics, Metabolism, Pharma- ceutics, and Toxicology}

by Chao Han, Charles B. Davis, and Binghe Wang, (Eds.): Wiley Hoboken, NJ, USA 2009, ix + 289,

ISBN 978-0-470-04491-9.

Price: EUR 89.99, USD 99.95

The laborious and hugely expensive process of taking a lead compound and attempting to develop it into a marketable medicine grows ever more complex and expensive as pharmaceutical companies struggle to develop new products, or replace products as they are released from patent protection. Scientific and technological advancements may open up new roads for drug development, but public perceptions of the industry are often suspicious at best, and made worse by high profile drug recalls as unfavourable safety data inevitably mount up. This book, largely written by scientists working in the pharmaceutical industry, attempts to provide an insight into how drug development can be improved in the early stages by integrating the critical areas in drug design, to minimise costs and drop-out, and more importantly improve safety and efficacy of the final product.

The book is divided into three parts (missing from the contents pages - perhaps to encourage integrated thinking?), concerning pharmacokinetics and metabolism, pharmaceutics, and predictive safety assessment. The chapters (2-7) of the first part deal with the experimental strategies currently used by pharmaceutical scientists to screen potential drug candidates for characteristics of absorption, distribution, metabolism and elimination (known as ADME). Following a very useful overview of pharmacokinetics in Chapter 2, the major physiological factors affecting the journey of a drug from administration to target site are covered in successive chapters, and the authors describe mechanisms of such processes in some detail without bogging the reader down too heavily. The chapter on cytochrome P450, the mention of which usually sends students screaming from the lecture theatre, is particularly detailed, but necessarily so to become meaningful as a useful reference source. Details of experimental assays are also included, with illustrative examples. A point well made in the conclusion of Chapter 5 is that many very useful drugs we have today would not meet the current stringent pharmacokinetic criteria for further development and their potential would never have been discovered.

Part 2 consists of a single but important chapter, on the integration of pharmaceutics into early drug development. The pharmaceutical industry may spend a lot of time and money on a lead compound, only to find at a late stage that the physicochemical properties of the compound severely hinder further development as a drug. This chapter serves as a reminder of the importance of considering pharmaceutics in drug design programmes.

Part 3 is concerned with safety, and discusses the need for better integration of toxicological studies early in the development of a lead compound. It focuses on the major causes of attrition (cardiotoxicity, hepatotoxicity, teratogenicity $\ldots$ ) and describes different assays and experiments which might aid in predicting these effects, preferably via high-throughput screening. Perhaps unsurprisingly, electrophysiological screening appears high on the list, and an entire chapter is dedicated to the importance of drug-induced long QT syndrome and its complications. Problematically for the industry, patchclamping is a highly specialised technique and attempts to automate for highthroughput screening remain far from perfect.

As a whole the book is text-heavy, but diagrams and illustrations, where they exist, are complementary. All of the chapters cite many recent references, and the authors appear well aware of the limitations of the various assays and strategies discussed. This is a useful reference source for those new to, or aspiring to join the pharmaceutical industry, for academics, and for students of pharmacy and pharmaceutical sciences.

N.C. Henney 